05

\title{
Твердотельный изгибно-вибрационный датчик низкочастотного магнитного поля на основе пьезоэлектрического эффекта
}

\author{
(C) А.Е. Панич, ${ }^{2}$ Г.С. Радченко, ${ }^{1}$ А.В. Скрылев, ${ }^{2}$ А.А. Панич, ${ }^{2}$ А.Ю. Малыхин ${ }^{2}$ \\ ${ }^{1}$ Южный федеральный университет, \\ 344022 Ростов-на-Дону, Россия \\ 2 Южный федеральный университет, Институт высоких технологий и пьезотехники, \\ 344090 Ростов-на-Дону, Россия \\ e-mail: grig1980@mail.ru
}

(Поступило в Редакцию 9 мая 2018 г.)

Произведен теоретический расчет оптимальных геометрических и электрофизических параметров и экспериментальное исследование эффективного отклика твердотельного гибридного датчика „магниткарбон-пьезоэлектрик“. Воздействием являлось внешнее магнитное поле низких частот изгибных резонансов от 1 до $230 \mathrm{~Hz}$. На основе теоретических расчетов создан экспериментальный прототип прибора. Построена частотная зависимость прямого пьезоэлектрического отклика на приложенное малосигнальное переменное магнитное воздействие.

DOI: $10.21883 / J T F .2019 .02 .47071 .180-18$

\section{Введение}

Магнитное поле как физический объект характерно силовым влиянием на движущиеся отдельные заряженные частицы или электрические токи. Справедлив и обратный эффект - наведение магнитного поля движущимися зарядами или токами. Эти эффекты в твердых телах с магнитной структурой неразрывно связаны с наличием у них собственной индуктивности, пропорционально которой находится магнитный поток. Именно этот параметр является ответственным за величину и направление возникающего на выходе устройства электромагнитного отклика [1-4]. В связи с этим для намагничивания возможны две принципиально противоположные ситуации: намагничивание против поля, которое подчиняется закону Фарадея, и твердотельное намагничивание по полю, которое слабо проявляется в парамагнетиках и намного более сильно в ферромагнетиках. Ясно, что прямая связь между электромагнитным откликом и воздействием может давать нежелательные погрешности в процессе измерения. Например, ферромагнитный образец подвержен возникновению фарадеевских по своей природе токов Фуко, которые препятствуют его намагничиванию. Также помещение в измерительную катушку для усиления индуктивности любого твердотельного материала резко изменяет эффективный отклик прибора в соответствии с конкретной твердотельной магнитной структурой вносимого вещества. Данная структура, как правило, очень сложна и часто бывает неизвестной, что затрудняет согласование электромагнитной выходной цепи с нагрузкой. Поэтому полностью разделить входную и выходную цепь электромагнитного или магнитострикционного преобразователя является невозможным. Равно как является невозможным полное погашение квазистатического магнитного воздействия в произвольной точке пространства в от- личие от аналогичного воздействия электростатической природы. В связи с этим перспективным направлением проектирования датчиков магнитных воздействий является использование кросс-эффектов пьезоэлектричества и магнитострикции, а также этих эффектов в отдельности [1-16]. В этом случае входная и выходная цепи связаны лишь механически, а именно через обратимый упругий отклик [10-16]. Известно, что скорость распространения упругих взаимодействий намного меньше, чем электромагнитных. Поэтому намного меньше становятся и нежелательные фазовые сдвиги, и искажения, и форма выходного сигнала остается практически той же, что имеется на входе. Иными перспективными путями для детектирования подобного воздействия и электромагнитного разделения входной и выходной цепи являются твердотельные электронные приборы Холла [17], сверхпроводящие датчики [18], и, в общем случае, использование электронной структуры. В этом случае главным фактором, ответственным за вышеописанное разделение, является быстрота передачи сигнала из входной цепи в выходную развязку, что эффективно нивелирует медленные, инерционные и механические помехи. В общем случае температурнозависимые полупроводниковые и даже проводниковые приборы на основе эффекта Холла [17] являются очень чувствительными и эффективными устройствами, но они для своей безотказной работы требуют существования стабильного электронного потока (или тока). Это условие может нарушаться в случае сильной радиации, температурных и электрических воздействий и других неблагоприятных внешних условий. Поэтому для случая необходимости не слишком быстрой передачи взаимодействий упругая связь может выступить в более перспективной роли. В каждом конкретном случае все зависит от надлежащего типа инженерной проблемы, что диктует необходимость как быстрой, так и медленной 
развязки входного и выходного сигналов. В настоящей работе предлагается низкочастотный прибор, в котором механические воздействия играют определяющую роль в детектировании слабых электромагнитных полей и их последующем усилении. Особый акцент здесь делается на конструкционных и расчетных особенностях магнитоэлектрического отклика прибора. Также в настоящей работе производятся вычисления и экспериментальное определение важных для практики технологических параметров.

\section{Теоретическое исследование}

Рассматривается сенсор, впервые описанный в работах $[5,11-16]$ (рис. 1 из $[5,15,16])$ в стандартных координатах Оxyz. Под действием приложенного магнитного поля в магните прибора возникает механический момент. Это приводит к изгибным колебаниям структуры. Край прибора, который зажат и свободен от магнита, совместим с началом координат, ось $x$ направим по длине, ось $y-$ по ширине, а ось $z$ по толщине структуры. Начало координат выберем на левом, жестко зажатом краю металлической или карбоновой пластины. Дифференциальным уравнением изгибных колебаний для гармонически меняющейся во времени деформации будет (1) $[6-10,14,16]$ :

$$
\frac{\partial^{4} z}{\partial x^{4}}+\frac{\rho^{*} S^{*}}{Y^{*} J_{y}^{*}} \frac{\partial^{2} z}{\partial t^{2}}=0 .
$$

Здесь $z-$ величина изгибной деформации, $t-$ время, $\rho^{*}, S^{*}, Y^{*}$ и $J_{y}^{*}$ - эффективные плотность, площадь, модуль Юнга и осевой момент инерции поперечного сечения структуры. Исходя из геометрии поперечного сечения в [6], эффективный осевой момент инерции сенсора будет равен $J_{y}^{*}=2 \frac{h_{p}^{3} w_{p}}{3}+\frac{h_{m}^{2} w_{p} h_{p}}{2}+\frac{h_{m}^{3} w_{m}}{12}$. Здесь $h$ и $w$ есть толщины и ширины металлической (карбоновой) пластины (нижний индекс $m$ ) и пьезоэлектрических слоев (нижний индекс $p$ ) соответственно. Эффективный модуль Юнга вычисляется методом усреднения как композитный материал связности $2-2$ по известным моделям, например, $[1,4]$.

Решением уравнения (1) является функция (2) продольной координаты $x$ и времени $t$ :

$$
\begin{aligned}
z(x, t) & =(A \sin (k k \cdot x)+B \cos (k k \cdot x)+C \operatorname{sh}(k k \cdot x) \\
& +D \operatorname{ch}(k k \cdot x)) \sin (\omega x)
\end{aligned}
$$

Величина $k k$ является фазовым множителем пространственной деформации и равна $\left(\frac{\rho^{*} S^{*}}{Y^{*} J_{y}^{*}}\right) \omega^{0.5}$. Также в (2) $A, B, C$ и $D$ есть константы, которые подлежат определению из граничных условий, и находятся нами далее. Также $\omega=2 \pi f$ есть круговая частота, $f$ есть стандартная частота детектируемого внешнего магнитного поля в единицах Hz.
Исходя из геометрии [5], граничными условиями для (2) являются следующие соотношения, находящие отражение в формулах (3)-(11) ниже:

1) при $x=0: z_{x=0}=0 ; \frac{d z}{d x_{x=0}}=0$ (условие отсутствия деформации и углового отклонения свободного от магнита края прибора, $z(x, t)$ есть поперечный прогиб в зависимости от времени и координаты);

2) при $x=l_{m} \quad\left(l_{m}\right.$ есть длина карбоновой базыоснования): $\frac{d^{2} z}{d x^{2}}=\frac{J V H}{Y^{*} J_{v}^{*}}$ (связь кривизны структуры с механическим моментом согласно теории сопротивления материалов); $\frac{d^{3} z}{d x^{3}}=0$ (условие отсутствия поперечных динамических силовых воздействий [5,7-10]).

Здесь $J$ - намагниченность магнита, $V$ - геометрический объем магнита в $\mathrm{m}^{3}, H$ есть амплитуда величины приложенного магнитного поля в единицах $\mathrm{A} / \mathrm{m}$. Из граничных условий получается решение для функции $z(x, t)$. Для зажатой с левого края пластины дисперсионное уравнение будет выглядеть так (3) по [14].

$$
\operatorname{ch}\left(k k \cdot l_{m}\right) \cos \left(k k \cdot l_{m}\right)=-1 \text {. }
$$

Наименьший корень данного уравнения $k k \cdot l_{m}=1.875$, найденный из графического решения трансцендентного уравнения, соответствует частоте первого резонанса, которая берется из эксперимента. Из этого соотношения определяется эффективная величина $\frac{\rho^{*} S^{*}}{Y^{*} J_{y}^{*}}$ для всей структуры по соотношению (9) ниже.

Уравнения пьезоэффекта могут быть записаны следующим образом:

$$
\begin{aligned}
& S_{1}(x)=T_{1}(x) / Y^{*}+d_{31} E_{3}(x), \\
& D_{3}(x)=d_{31} T_{1}(x)+\varepsilon_{33} E_{3}(x) .
\end{aligned}
$$

Здесь $S_{1}(x, Z, t)=-\frac{d^{2} z(x, t)}{d x^{2}} Z$ есть кривизна сенсора, функции $T_{1}(x)$ и $E_{3}(x)$ являются генерируемым механическим напряжением и поперечным электрическим полем, $d_{31}$ и $\varepsilon_{33}$ есть пьезомодуль и диэлектрическая проницаемость пьезоэлектрика, $Z$ есть величина поперечной координаты (не путать с прогибом $z(x, t))$. Граничным условием электростатики [5,14-16] является равенство нулю электрической индукции на границе раздела между пьезоэлектриком и воздухом $\left.D_{3}\right|_{Z=h_{p}}=0$ [5] и во всем объеме. Подставляя в (4) $z(x, t)$ и усредняя по координате $x$, получаем базовое соотношение (5) для эффективного генерируемого напряжения. Значение производной $\frac{d z}{d x}\left(l_{p}\right)$ на краю пьезоэлектрика является угловым отклонением его правой крайней точки и в работе [16], где также строится описание данного датчика, оно задавалось экспериментально. Ясно, что это гораздо более трудоемко, так как требует наличие и применение чувствительного микрометра, в отличие от развиваемого в настоящей работе метода вычислений, основанного на экспериментальном определении частоты основного тона для определения оптимальных размеров прибора. Этот факт также делает невозможным непосредственное сравнение теории [16] с результатами по формулам (7), (9) ниже, однако если из эксперимента известно 
угловое отклонение края пьезоэлектрика, то для сравнения с [16] применимо соотношение (5), которое также приводится в [14]:

$$
U=-\frac{2 d_{31} Y^{*}\left(h_{p}\right)^{2}}{l_{p} \varepsilon_{33}\left(1-\left(k_{31}\right)^{2}\right)} \int_{0}^{l_{p}} \frac{d^{2} z}{d x^{2}} d x .
$$

В случае равных длин металла (карбона) и пьезоэлектрика параметр $l_{p}$ меняется на общую длину $l$ во всех соотношениях ниже, и для теоретических расчетов используется соотношение (11) настоящей работы. Здесь $k_{31}=\left(d_{31}\right)^{2} \frac{Y^{*}}{\varepsilon_{33}}$ есть квадрат электромеханической связи выходной твердотельной цепи „пьезоэлектрик-карбон“. В процессе теоретического прогнозирования и экспериментального изготовления композита „пьезоэлектрик-карбон“ необходимо контролировать данный параметр с целью его наибольшего увеличения. Следовательно, в качестве пластиныоснования подходят составляющие с максимально возможными модулями Юнга. Однако дальнейшее увеличение данного параметра приводит к усилению роли влияния высших мод и повышению резонансной частоты основной (первой) моды, что не всегда подходит для конкретной технической реализации прибора и цели измерения. Увеличение же объемной плотности, равно как и площади поперечного сечения структуры напротив приводит к уменьшению характерных резонансных частот на итоговой АЧХ. Рост осевого момента инерции приводит к росту резонансных частот и уменьшению амплитуды колебаний.

Аналитические формулы для найденных констант и итогового напряжения получены в настоящей работе впервые и могут быть записаны в следующем виде $(6),(7),(11)$ :

$$
\begin{aligned}
A & =\frac{1}{2 k k^{2} J_{y}^{*} Y^{*}} \frac{\operatorname{sh}\left(k k \cdot l_{m}\right)-\sin \left(k k \cdot l_{m}\right)}{1+\cos \left(k k \cdot l_{m}\right) \operatorname{ch}\left(k k \cdot l_{m}\right)} J V H, \\
B & =\frac{1}{2 k k^{2} J_{y}^{*} Y^{*}} \frac{\operatorname{ch}\left(k k \cdot l_{m}\right)+\cos \left(k k \cdot l_{m}\right)}{1+\cos \left(k k \cdot l_{m}\right) \operatorname{ch}\left(k k \cdot l_{m}\right)} J V H, \\
C & =\frac{1}{2 k k^{2} J_{y}^{*} Y^{*}} \frac{\operatorname{sh}\left(k k \cdot l_{m}\right)-\sin \left(k k \cdot l_{m}\right)}{1+\cos \left(k k \cdot l_{m}\right) \operatorname{ch}\left(k k \cdot l_{m}\right)} J V H, \\
D & =\frac{1}{2 k k^{2} J_{y}^{*} Y^{*}} \frac{\operatorname{ch}\left(k k \cdot l_{m}\right)+\cos \left(k k \cdot l_{m}\right)}{1+\cos \left(k k \cdot l_{m}\right) \operatorname{ch}\left(k k \cdot l_{m}\right)} J V H .
\end{aligned}
$$

В полном соответствии с найденным ранее дисперсионным соотношением константы имеют экстремум при выполнении условия (3) и обладают в знаменателе квадратом фазового множителя. Итоговая формула для генерируемого напряжения может быть записана в виде (7), с полным учетом соотношений (5), (6):

$$
\begin{aligned}
& U=\frac{d_{31}\left(h_{p}\right)^{2} J V H}{k k \cdot J_{y}^{*} l_{p} \varepsilon_{33}\left(1-\left(k_{31}\right)^{2}\right)} \\
& \sin \left(k k\left(l_{p}-l_{m}\right)\right)+\operatorname{ch}\left(k k \cdot l_{p}\right)\left(\sin \left(k k \cdot l_{m}\right)\right. \\
& \times\left(\frac{\left.-\operatorname{sh}\left(k k \cdot l_{m}\right)\right)+\cos \left(k k \cdot l_{p}\right) \operatorname{sh}\left(k k \cdot l_{m}\right)}{1+\cos \left(k k \cdot l_{m}\right) \operatorname{ch}\left(k k \cdot l_{m}\right)}\right. \\
& \cos \left(k k \cdot l_{m}\right) \operatorname{sh}\left(k k \cdot l_{p}\right) \\
& \left.+\frac{+\operatorname{ch}\left(k k \cdot l_{m}\right)\left(\sin \left(k k \cdot l_{p}\right)+\operatorname{sh}\left(k k \cdot l_{p}\right)\right)}{1+\cos \left(k k \cdot l_{m}\right) \operatorname{ch}\left(k k \cdot l_{m}\right)}\right) \text {. }
\end{aligned}
$$

Коэффициент магнитоэлектрического преобразования определяется следующим образом (8):

$$
a_{E}=\frac{1}{\left(2 h_{p}+h_{m}\right)} \frac{U}{H} \text {. }
$$

Все результаты компьютерного моделирования производились в [14] согласно соотношению (7), а в настоящей работе для определения оптимальных размеров прибора согласно (11). Основным результатом являлось определение оптимальных толщин металла и пьезоэлектрика для получения наибольшего генерируемого напряжения, что ранее [14-16] сделано в литературе достаточно подробно. Однако до настоящего исследования не были применены карбоновые основания, что было сделано здесь впервые. Особенно важным является факт, что все зависимости $U$ от толщины как пьезоэлектрика, так и металла (карбона) должны быть немонотонными, с максимумом при определенной величине изменяемого геометрического параметра [14-16].

Эквивалентная жесткость структуры $b b$ определится так $b b=\frac{\rho^{*} S^{*}}{Y^{*} J_{*}^{*}}$. Тогда для ее численного определения получаем соотношение (9):

$$
b b=\frac{15}{8 l_{m}} \frac{1}{\sqrt{f_{1}}} .
$$

Здесь $f_{1}$ - это частота первого резонанса, определяемая экспериментально из анализа вибраций конкретной структуры. Далее получаем базовое соотношение (10) для волнового вектора структуры при неоднородной пространственной деформации, который входит во все соотношения выше:

$$
k k=b b \cdot \sqrt{f} .
$$

Особую важность представляет случай, когда длина керамического компонента равна длине карбоновой пластины. Для этого случая перейдем в формуле (7) к предельному случаю $l_{m}=l_{p}=l$. В этом важном для практики случае для итогового расчета эффективного ге- 
нерируемого напряжения получается соотношение (11):

$$
\begin{aligned}
U & =\frac{2 d_{31}\left(h_{p}\right)^{2}}{k k \cdot J_{y}^{*} l \varepsilon_{33}\left(1-\left(k_{31}\right)^{2}\right)} \\
& \times\left(\frac{\operatorname{ch}(k k \cdot l) \sin (k k \cdot l)+\cos (k k \cdot l) \operatorname{sh}(k k \cdot l)}{(1+\cos (k k \cdot l) \operatorname{ch}(k k \cdot l)}\right) J V H .
\end{aligned}
$$

Важно отметить, что для случая равных длин дисперсионное соотношение сохраняет свою форму, число возможных численных решений и их величину.

Основным отличием настоящей теории от [16] является предположение о том, что вся электрическая индукция в образце равна нулю, в [16] же она обладает пространственными вариациями и в граничном условии нивелируется лишь ее интегральная величина, что не является оправданным для тонких слоев пьезокерамики.

\section{Экспериментальное исследование}

Экспериментальное исследование осуществлялось с помощью составной гибридной структуры „магниткарбон-пьезоэлектрик“ (рис. 1) в магнитном поле амплитудой $0.05 \mathrm{Oe}$. Карбоновая пластинка изготавливалась методом укладки слоев карбона в эпоксидной смоле для получения итоговым объектом наибольшего модуля Юнга. На карбоновую пластинку были нанесены методом склеивания два поляризованных вовне керамических слоя из керамики ПКП-12. Левый конец итоговой конструкции симметричного биморфа был жестко зажат. На правом конце структуры располагался неодимовый магнит (рис. 1) с остаточной намагниченностью, равной 1.4 Т. Для измерений использовался модуль измерения температурных и частотных характеристик, изготовленный в МТУ МИРЭА (г. Москва). Магнитное поле разной частоты создавалось встроенными в установку катушками Гельмгольца.

Геометрические размеры составных частей были следующими:

1. Карбоновая пластина-основание $-26 \times 1.8 \times 0.2 \mathrm{~mm}^{3}$.

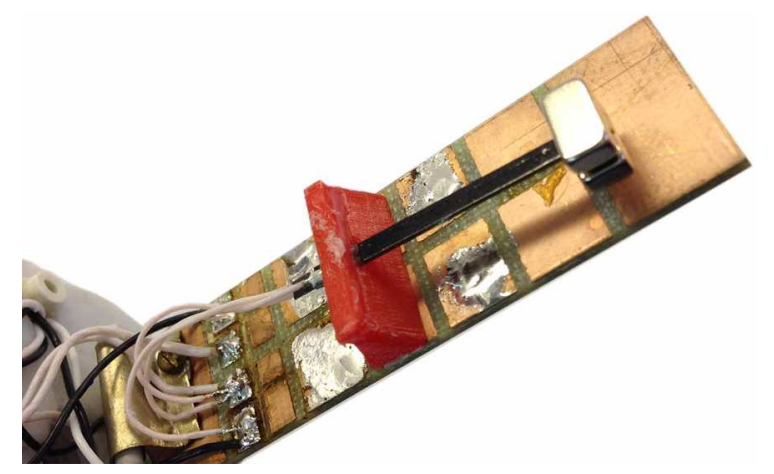

Рис. 1. Фотография (внешний вид) симметричного датчика магнитного поля „магнит-карбон-пьезоэлектрик“.

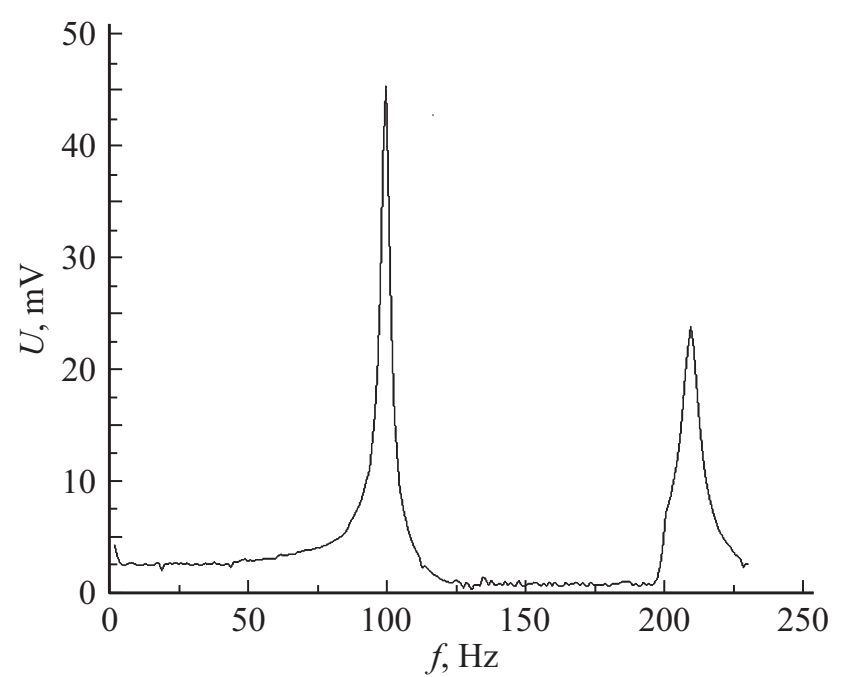

Рис. 2. Экспериментально определенная частотная зависимость амплитуды генерируемого напряжения от переменного магнитного поля величиной 0.05 Ое симметричного биморфа „магнит-карбон-пьезоэлектрик“.

\section{2. Пьезокерамика ПКП-12 $-26 \times 1.8 \times 0.2 \mathrm{~mm}^{3}$.}

3. Неодимовый магнит $-4 \times 7 \times 3 \mathrm{~mm}^{3}$.

Все геометрические параметры были подобраны в результате прогнозирования оптимального отклика по генерируемому напряжению в условиях разомкнутой цепи по формуле (11), зная плотности и модули Юнга составных частей биморфа. Для расчета использовались следующие значения: для пьезокерамики ПКП-12, производимой в НТКБ „Пьезоприбор“ ЮФУ - относительная диэлектрическая проницаемость $\varepsilon_{33}^{T} / \varepsilon_{0}=4600$, пьезомодуль $d_{31}=310 \mathrm{pC} / \mathrm{N}$, модуль Юнга $c_{11}^{E}=127 \mathrm{GPa}$, плотность $\rho=7500 \mathrm{~kg} / \mathrm{m}^{3}$, карбонового основания $c=230 \mathrm{GPa}, \rho=1500 \mathrm{\kappa r} / \mathrm{m}^{3}$. Результаты эксперимента на серии образцов показали хорошее соответствие с амплитудным значением выражения (10).

Магнитное поле с частотой от 1 до $230 \mathrm{~Hz}$ вызывало изгибные колебания карбона и пьезоэлектрика, и в результате прямого пьезоэффекта на серебряных электродах генерировалось электрическое напряжение. Его последующее усиление и обработка не производились. Все измерения осуществлялись при комнатной температуре с шагом по частоте, равным $1 \mathrm{~Hz}$, вручную и записывались в таблицу Excel, по которой затем был построен рис. 2. Все измерения проводились без приложения постоянного подмагничивающего поля, что является важным при практических применениях данной составной конструкции. При измерениях прибор был в вертикальном положении.

На рис. 2 ниже изображена частотная зависимость генерируемого напряжения от частоты приложенного магнитного поля. Хорошо видно, что в низкочастотной области отклик стабилен по частоте и достаточен для усиления и обработки. Два явно выраженных резонанса наблюдаются на частотах 100 и $210 \mathrm{~Hz}$. В частотной 
области между резонансами отклик достаточно слабый. На кривой имеются незначительные флуктуации, которые, видимо, связаны с вихревыми токами снаружи измерительной камеры и магнитным полем Земли. В резонансной области наблюдается хорошая чувствительность датчика к внешнему магнитному воздействию, достаточная для широкого практического применения после усиления и обработки генерируемого сигнала. В ходе проведенного эксперимента получен магнитоэлектрический отклик свыше $1 \mathrm{~V} / \mathrm{Oe}$ в резонансном режиме измерения. Совокупность теоретических данных указывает на изгибную природу обоих полученных резонансных пиков выходного сигнала.

Для конкретного применения формул (7),(11) для построения расчетной частотной зависимости генерируемого напряжения аналогично [14] необходимо знать частоту первого резонанса твердотельной структуры [5] и коэффициент изгибного затухания. Оба эти параметра могут быть найдены лишь эмпирически, их точное определение наталкивается на трудности, поэтому такие и подобные им зависимости в настоящей работе не строились. Корректное решение данной проблемы будет предметом дальнейших исследований, имеющих в своей основе теорию настоящей работы.

\section{Заключение}

В работе теоретически и экспериментально исследован симметричный изгибно-вибрационный биморф на основе карбона, дополненный магнитом для измерения и детектирования низкочастотного магнитного поля. Также данный датчик обладает чувствительностью к любым электромагнитным воздействиям, содержащим значительную по амплитуде магнитную составляющую.

Как видно из основных расчетных формул, эффективный отклик по напряжению прямо пропорционален детектируемому магнитному полю. Причем в базовые уравнения не входят пространственные вариации магнитного поля и его временные зависимости. Это говорит о том, что данный прибор должен работать на очень низких частотах с несколько большей эффективностью, чем приборы на основе эффекта Фарадея или сверхпроводников [18]. В самом деле, из дифференциального уравнения Максвелла для гармонического воздействия следует очевидное соотношение $\operatorname{rot} \mathbf{E}=-\frac{\partial \mathbf{B}}{\partial t}=-\omega \mathbf{B}$, т.е. эффективный электрический отклик пропорционален частоте внешнего воздействия, имеет фарадеевский механизм возникновения и меняется с координатой, имея поворот по фазе. Этих недостатков практически лишен настоящий прибор, потому что возникающее напряжение априори является вполне измеряемой интегральной величиной, синфазной с внешним воздействием, а также отсутствует пропорциональная зависимость от частоты.

Негативным влиянием в случае возможного применения датчика в реальных условиях могут быть вибрации, внешние толчки и очень сильные внешние нагрузки. Эти влияния в отличие, например, от сильной радиации, могут быть устранены методами визуального контроля и применением низкочастотных резонансных фильтров, а также механических демпферов. Также к преимуществам такого прибора можно отнести линейность его полевых характеристик в ответ на внешнее воздействие, в отличие от датчиков Холла [17] и сверхпроводящих датчиков в сильных полях [18] при неблагоприятных воздействиях. Поэтому развитие подобных пьезомагнитных сенсоров на основе упругих кросс-эффектов является перспективным направлением развития технологии измерения внешних квазистатических магнитных полей.

Исследования проведены при финансовой поддержке Министерства образования и науки РФ в рамках реализации комплексного проекта „Разработка и создание высокотехнологичного производства мобильного гидроакустического комплекса освещения обстановки в различных акваториях Мирового океана на основе современных пьезоэлектрических средств нового поколения“ (Договор № 03.G25.31.0276 от 29.05.2017).

Работа выполнена с использованием оборудования ЦКП „Высокие технологии““ ЮФУ.

\section{Список литературы}

[1] Nan C.-W., Bichurin M.I., Dong S., Viehland D., Srinivasan G. // J. Appl. Phys. 2008. Vol. 103. P. 031101 (1-35).

[2] Филиппов Д.А., Галкина Т.А., Лалетин В.M., Srinivasan G. // Письма ЖТФ. 2012. Т. 38. Вып. 2. С. 82-86.

[3] Филиппов Д.А. // ФТТ. 2012. Т. 54. Вып. 6. С. 1112-1115.

[4] Petrov V.M., Srinivasan G., Bichurin M.I., Galkina T.A. // J. Appl. Phys. 2009. Vol. 105. P. 063911 (1-6).

[5] Liu G., Li X., Chen J., Shi H., Xiao W., Dong S. // Appl. Phys. Lett. 2012. Vol. 101. P. 142904 (1-4).

[6] Patil D.R., Chai Y., Kambale R.S., Jeon B.-G., Yoo K., Ryu J., Yoon W.-H., Park D.-S., Jeong D.-Y., Lee S.-G., Lee J., Nam J.H., Cho J.-H., Kim B.-I., Kim K.H. // Appl. Phys. Lett. 2013. Vol. 102. P. 062909 (1-4).

[7] Ballas R.G. Piezoelectric Multilayer beam bending actuators. Berlin-Heidelberg: Springer-Verlag, 2007. 353 p.

[8] Феодосьев В.И. Сопротивление материалов. М.: Наука, 1967. $552 \mathrm{c}$.

[9] Wu N., Wang Q., Quek S.T. // J. Sound and Vibration. 2010. Vol. 329. P. 1126-1136.

[10] Radchenko G.S. // J. Phys. D: Appl. Phys. 2008. Vol. 41. P. 055421 (1-4).

[11] Smits Jan G., Dalke S.I., Cooney T.K. // Sensors and Actuators. A. 1991. Vol. 28. P. 41-61.

[12] Wang Q.-M., Du X.-h., Xu B., Cross L.E. // J. Appl. Phys. 1999. Vol. 85. P. $1702-1712$.

[13] Wang Q.-M., Cross L.E. // Ferroelectrics. 1998. Vol. 215. P. 187-213. 
[14] Радченко Г.С., Радченко М.Г. // Письма ЖТФ. 2014. Т. 40. Вып. 12. С. 19-23.

[15] Радченко Г.С., Радченко М.Г. // ЖТФ. 2014. Т. 84. Вып. 10. C. $39-43$.

[16] Liu G., Dong S. // J. Appl. Phys. 2014. Vol. 115. P. 084112 (1-8).

[17] Веселов А.Г., Рябушкин С.Л., Шуллер И.Я. // ЖТФ. 1994.

T. 64. Вып. 11. С. 204-206.

[18] Черных С.В. // ЖТФ. 2013. Т. 83. Вып. 2. С. 129-135. 